\title{
Quantum entanglement, indistinguishability, and the absent-minded driver's problem
}

\author{
Adán Cabello \\ Departamento de Física Aplicada II, Universidad de Sevilla, E-41012 Sevilla, \\ Spain \\ John Calsamiglia \\ Institut für Theoretische Physik, Universität Innsbruck, A-6020 Innsbruck, \\ Austria
}

\begin{abstract}
The absent-minded driver's problem illustrates that probabilistic strategies can give higher pay-offs than deterministic ones. We show that there are strategies using quantum entangled states that give even higher pay-offs, both for the original problem and for the generalized version with an arbitrary number of intersections and any possible set of pay-offs.
\end{abstract}

Key words: Quantum games, Entanglement and quantum non-locality PACS: 03.65.Ud, 02.50.Le

\section{Introduction}

\subsection{The absent-minded driver's problem}

The so-called paradox of the absent-minded driver was introduced by Piccione and Rubinstein in [1] and further discussed in [2,3] and references therein: an individual is sitting late at night in a bar planning his midnight trip home. The trip starts at the bar, the START in Fig. 1. There is a highway with two consecutive exits (or intersections), $X$ and $Y$, and he has to take the

Email addresses: adan@us.es (Adán Cabello), John.Calsamiglia@uibk.ac.at (John Calsamiglia).

Preprint submitted to Elsevier Science 9 June 2018 
second, $Y$, to get home (pay-off 4). If he takes the first one, he arrives at a bad neighborhood (pay-off 0), and if he fails to take either, he has to stay in a motel at the end of the highway (pay-off 1). He cannot go back. There are two essential assumptions:

(I) Indistinguishability: The intersections $X$ and $Y$ are indistinguishable by any experiment performed at one intersection. When the driver is at one intersection, no experiment can give him information about which intersection he is at.

(II) Absent-mindedness: The driver is absent-minded and is aware of this fact. Absent-mindedness only affects his memories about whether he has already gone through one of the intersections; at $X$ he knows he might be at $Y$ but has forgotten passing $X$, and at $Y$ he cannot remember passing $X$. Apart from this, the driver is perfectly able to remember anything else.

Some remarks about these assumptions follow:

(i) These assumptions are not independent: the indistinguishability of the intersections is only relevant for an absent-minded driver, and absent-mindedness is only relevant when the intersections are indistinguishable (otherwise, the driver could obtain information for decision-making in spite of his absentmindedness).

(ii) Since the driver's absent-mindedness is limited to his memories about the intersections, but does not prevent him from possessing information about the rest of the universe, then it is forbidden any experiment at one intersection whose result, together with any information about the rest of the universe, allows the driver to obtain information about which intersection he is at.

(iii) Implicit in the rules is the fact that the driver cannot transmit information from one intersection to the rest of the universe (and, in particular, to the other intersection), because this could be used to distinguish the intersections.

The above scenario allows Piccione and Rubinstein to exhibit a conflict between two ways of reasoning at an intersection:

"Planning his trip at the bar, the decision maker must conclude that it is impossible for him to get home and that he should not exit when reaching an intersection. Thus, his optimal plan will lead him to spend the night at the motel and yield a payoff of 1 . Now suppose that he reaches an intersection. If he had decided to exit, he would have concluded that he is at the first intersection. Having chosen the strategy to continue, he concludes that he is at the first intersection with probability $1 / 2$. Then, reviewing his plan, he finds that it is optimal for him to leave the highway since it yields an expected payoff of 2. Despite no new information and no change in his 


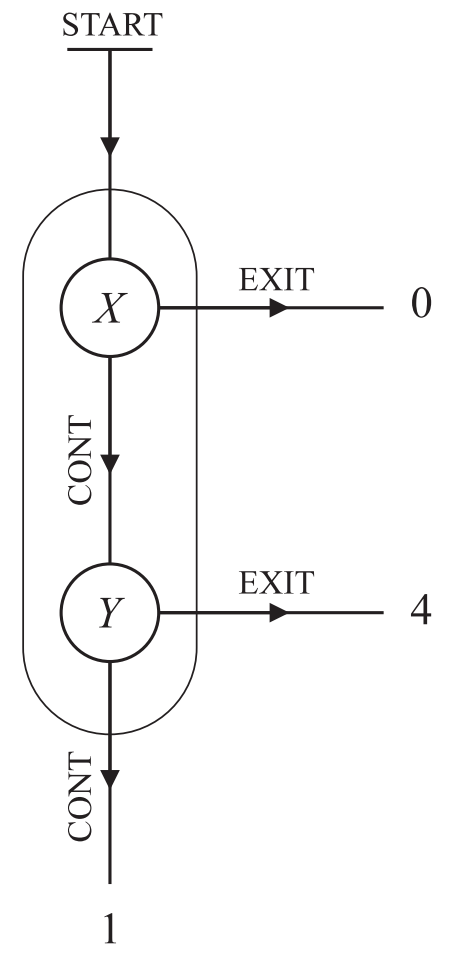

Fig. 1. The absent-minded driver's problem.

preferences, the decision maker would like to change his initial plan once he reaches an intersection! [1]"

Piccione and Rubinstein make use of this apparent paradox to illustrate the advantages of probabilistic (or random [1], or mixed) strategies versus deterministic (or pure [1]) strategies. At the intersections, the driver can either CONTINUE along or EXIT the highway. Accordingly, there are are two possible deterministic strategies: either to always CONTINUE (pay-off 1) or to always EXIT (pay-off 0). Alternatively, at the intersections, the driver can toss a (suitable weighted) coin with a probability $p$ for heads (which means CONTINUE) and a probability $1-p$ for tails (which means ExIT). The expected pay-off of this probabilistic strategy is $4 p(1-p)+p^{2}$. Therefore, if $p>1 / 3$, this strategy gives a higher pay-off than the best deterministic strategy. The optimal probabilistic strategy consists of choosing $p=2 / 3$ (pay-off $4 / 3$ ).

\subsection{Quantum strategies}

Game theory has recently found a new direction based on the possibility of the resources of quantum mechanics becoming available to the players [4]. This introduces new possibilities which lead to advantages over their classical counterparts (however, see [5]). So far, the advantages of the so-called quantum strategies have been limited to situations involving players with per- 
fect recall $[6,7,8,9,10,11,12]$. As we shall illustrate, decision problems involving players with imperfect recall are a promising arena for the application of quantum resources. In particular, we will see that quantum mechanics allows the absent-minded driver to make use of correlations without compromising the assumptions (or rules) of the problem and thereby obtain higher pay-offs.

\section{Physical realization of probabilistic strategies}

Let us start by re-examining from the perspective of physics what Piccione and Rubinstein consider the optimal solution to the absent-minded driver's problem: a probabilistic strategy. Can we implement a probabilistic strategy in a real experiment without compromising the assumptions of the problem at a fundamental level? In particular, how can we accomplish the driver's action of "tossing a coin" in the intersection satisfying the requirement of even-in-principle indistinguishability of the intersections? Strictly speaking, the driver is not allowed to carry a coin since he could use it to bypass his absentmindedness and keep track of the intersections he passes. However, the driver may instead place a coin in each of the intersections before the trip starts.

The first problem is which physical system can be used as a coin. Strictly speaking, real coins are highly complex systems and therefore, in principle, it is not difficult to distinguish between two of them. This problem can be eluded by replacing real coins by identical physical systems (i.e., those with the same composition of identical elementary particles and prepared in the same physical state).

The next problem is the mechanism for "tossing" the coin. By "tossing a coin" we mean that the driver can use his coin to perform a two-output experiment with probability $p$ for one of the outputs. However, this is a very difficult task from the perspective of classical physics, where the results of experiments are essentially predefined in the state of the coin and the device used to toss it. In this scenario both are under the driver's control, and therefore the result of the experiment can in principle be fixed by the driver beforehand. In quantum mechanics, however, it can be proven, under some general assumptions, that the results of certain experiments are not predefined [13,14], but are created [15] when the experiment is performed. Therefore, at least from the perspective of quantum mechanics, there is a method for tossing a coin without compromising the assumptions of the absent-minded driver's problem at a fundamental level. The driver can place a "quantum coin" [6] or qubit (i.e., a two-level

quantum system), say a spin- $\frac{1}{2}$ particle, in each of the intersections ( $X$ and $Y)$. Let us denote the quantum state of the two quantum coins as $\rho_{X Y}$. 
The indistinguishability assumption implies that the quantum state of each quantum coin is represented by the same reduced density matrix, $\operatorname{tr}_{X} \rho_{X Y}=$ $\operatorname{tr}_{Y} \rho_{X Y}$. Moreover, states with unequal reduced density matrices hidden in classical mixtures (i.e., states of the form $\rho_{X Y}=\sum_{i} p_{i}\left|\psi_{X Y}^{i}\right\rangle\left\langle\psi_{X Y}^{i}\right|$, where, in some instances, $i=a$, the quantum coins are in different states, $\operatorname{tr}_{X} \psi_{X Y}^{a} \neq$ $\left.\operatorname{tr}_{Y} \psi_{X Y}^{a}\right)$ are forbidden, since there is no way to ensure that the driver does not have a priori knowledge on the particular instance that will appear in each intersection in a particular trip. This knowledge would allow the driver to learn, with a given probability, which intersection he is at. Hence this does not fulfill the assumption of indistinguishability.

A probabilistic strategy can be implemented right away by a suitable measurement on a suitable quantum state. For instance, by preparing the qubits in the pure state

$$
|\Phi\rangle=|\phi\rangle_{X}|\phi\rangle_{Y}
$$

where

$$
|\phi\rangle=\sqrt{p}|0\rangle+\sqrt{1-p}|1\rangle,
$$

and measuring at the intersection the observable

$$
\sigma_{z}=|0\rangle\langle 0|-| 1\rangle\langle 1|
$$

When the driver arrives to any intersection, he measures $\sigma_{z}$, and the probability of obtaining CONTINUE is $p$, and the probability of obtaining EXIT is $1-p$.

\section{Entanglement-assisted strategies}

At this point, one can think about the possibility of using more complex quantum mechanical resources instead of just experiments on quantum systems as generators of random numbers. Indeed, here we show that there are quantum strategies which give higher expected pay-offs than any probabilistic one. For instance, let us prepare two qubits in the singlet state

$$
|\Psi\rangle=\frac{1}{\sqrt{2}}(|01\rangle-|10\rangle),
$$

and put one qubit in each intersection. The reduced states of each qubit are both maximally mixed and cannot be described in terms of classical correla- 
tions [13]. When the driver arrives at the first intersection, he measures $\sigma_{z}$, and the probability of obtaining either CONTINUE or EXIT is $1 / 2$. However, by using the singlet state, the driver has managed to introduce correlations between both intersections without violating the assumptions of the absentminded driver's scenario: whenever he obtains CONTINUE in the first intersection, he obtains ExIT in the second, and vice-versa. Therefore, the expected pay-off is 2 , which is higher than the highest expected pay-off $(4 / 3)$ for a probabilistic strategy. Note, that as mentioned earlier, the use of pseudo-random (i.e., deterministic) procedures to generate anti-correlations would immediately lead to distinguishable intersections: in principle the driver could be in possession of the "seed" and would be able to recognize the intersections by the different outcomes of their pseudo-random number generators.

Moreover, the previous strategy achieves not only a higher pay-off than any probabilistic strategy, but actually the optimal pay-off for the absent-minded driver's problem. This can be proven as follows. The driver has complete knowledge of the state of the universe before the game starts, hence we can assume that the initial state is pure $|\Phi\rangle_{X Y R}$, where $R$ refers to the Hilbert space of the rest of the universe. During the game he will have restricted access to that state via sequential local operations on systems $X$ and $Y$. Moreover, the driver lacks any memory or reference that tells him which intersection he is at and therefore cannot choose a strategy accordingly. With all the above considerations we can now assume without loss of generality that an optimal strategy for the absent-minded driver will consist on preparing the quantum systems in $X Y$ in a pure state $|\Psi\rangle$ and, on his arrival at an intersection, performing a measurement in a fixed basis, let us say the computational basis $\{|0\rangle,|1\rangle\}$. Indeed, such a scheme will always be able to provide the same correlations between the measurement outcomes than the most general scheme, and therefore provide the optimal pay-off. We now have to maximize over the possible states $|\Psi\rangle=\sum_{i, j=0}^{1} \alpha_{i j}|i j\rangle$. Since the coherences do not affect the pay-off, it suffices to look at the probability distribution $\left\{\left|\alpha_{i j}\right|^{2}\right\}$ of distinct classical instruction sets $\{(i, j)\}$ and check for full compliance of the rules when the phases are picked. Any instruction that does not allow the driver to distinguish between the intersections can be expressed as a convex linear combination of three basic strategies. The first is the instruction to CONTINUE in both intersections (pay-off $\left.P_{1}=1\right)$, represented by $(0,0)$; the second is to EXIT in both intersections (pay-off $P_{2}=0$ ), represented by $(1,1)$; and the third gives CONTINUE in $X$ and Exit in $Y$ for half of the runs, and Exit in $X$ and Continue in $Y$ for the other half (pay-off $P_{3}=2$ ), and can be represented by $(0,1)+(1,0)$. The optimal strategy should maximize $P=\sum_{j=1}^{3} p_{j} P_{j}$, where the probabilities are positive, $p_{j} \geq 0$, and normalized, $\sum_{j=1}^{3} p_{i}=1$. Since the expected pay-off itself is a convex function, it is clear that its maximum value will be achieved with the extreme strategy of highest pay-off; that is, the optimal pay-off is $P=P_{3}=2$ and can be reached with the quantum state of Eq. (4). 


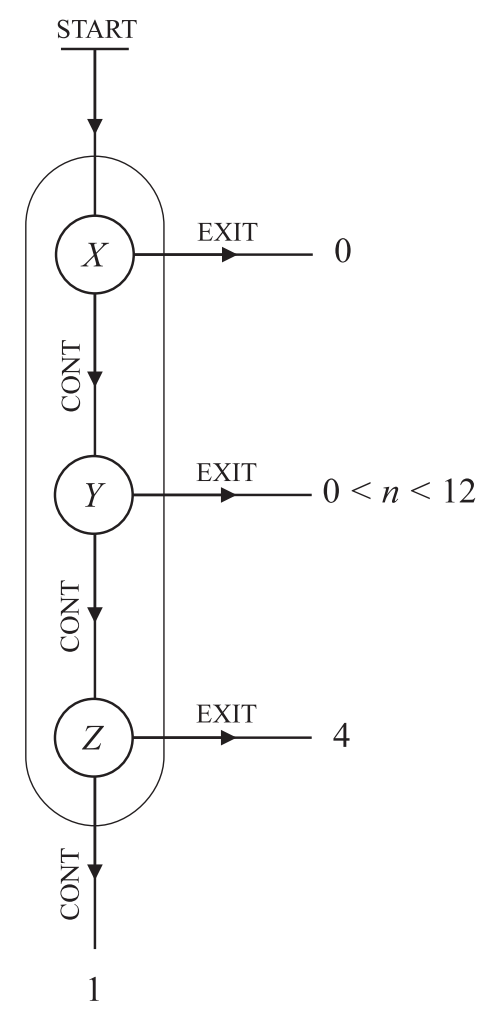

Fig. 2. The absent-minded driver's problem with three intersections.

A natural question is whether or not the same maximum pay-off could be achieved with less quantum resources. Can we mimic the strategy using the state (4) by means of a single qubit (as a source of genuine randomness) coupled to a classical mechanism to generate the desired correlations? The answer is no. On one hand, obtaining the maximum pay-off does not require perfect correlations but perfect anti-correlations between the results on both intersections, and any classical mechanism capable of generating different results would introduce a distinction between the intersections. On the other hand, such a classical mechanism could be used to transmit information between the intersections when the driver is inside one of them and thus violates (iii). Therefore we conclude that the strategy using two qubits in the state (4) is the simplest giving the maximum pay-off. At this point we also note that even if one could justify the use of pseudo-random number generators (or any other classical source of randomness), justifying the use of two generators that create correlated random outputs is much more demanding, as it immediately implies the existence of a reproducible and hence predictable mechanism of generating the outputs. 


\section{The absent-minded driver's problem with more intersections}

\subsection{An example with three intersections}

Let us now consider the absent-minded driver's problem with $N>2$ intersections. Here we can restrict ourselves to strategies where, at each intersection, the driver measures in the computational basis the prepared $N$-party pure state $|\Psi\rangle=\sum_{\vec{v}} \alpha_{\vec{v}}|\vec{v}\rangle$, where $\left\{\vec{v}_{i}\right\}_{1}^{d}$ is the set of $d=2^{N} N$-dimensional binary arrays, and corresponds to the set of instructions $\{(a, b, . N ., e)\}$. Here, finding the optimal strategy also requires finding the vertices of the convex polytope that results from imposing indistinguishability conditions on the simplex probability space $\left\{p_{1}=\left|\alpha_{v_{1}}\right|^{2}, \ldots, p_{d}=\left|\alpha_{v_{d}}\right|^{2}\right\}$. The condition for the indistinguishability of the intersections is that the reduced density matrices in each intersection have to be identical $\rho_{1}=\ldots=\rho_{N}$. In the computational basis, the diagonal matrix elements are equal if and only if all components of the vector $\vec{u}=\sum_{m} p_{m} \vec{v}_{m}$ are identical, i.e.,

$$
\vec{u}(i)=\vec{u}(j) \forall i, j=1, \ldots, 2^{N}
$$

For the moment we will disregard the conditions that arise from equating the out-of-diagonal terms of the reduced density matrices.

Any legitimate solution can be expressed as a convex combination of the solutions associated to each vertex of the polytope. Each vertex $E$ is specified

by a vector $\vec{p}^{E}$ in the probability space. As before, the optimal pay-off can be reached at one of the vertices, and the corresponding mixed set of instructions can be mapped into a quantum superposition by fixing the arbitrary phases. The extremal property of the vertices implies that all arrays contributing to a specific vertex, i.e., $\left\{\vec{v}_{m}: p_{m}^{E} \neq 0\right\}$ have to be linearly independent. Using this fact and the indistinguishability condition, it can be easily seen that two arrays contributing to a vertex cannot differ solely in a single component. That is, for any $(N-1)$-dimensional binary array $\vec{x},(1, \vec{x})$ and $(0, \vec{x})$ cannot appear together in the convex combination that defines a vertex. From this it follows that all the out-of-diagonal matrix elements of the reduced density operator $\rho_{1}=\operatorname{tr}_{2, \ldots, N} \rho^{E}$ have to vanish,

$$
\left\langle 1\left|\rho_{1}\right| 0\right\rangle=\sum_{\{\vec{x}\}}\left\langle 1 \vec{x}\left|\rho^{E}\right| 0 \vec{x}\right\rangle=0,
$$

where $\rho^{E}=\left|\phi^{E}\right\rangle\left\langle\phi^{E}\right|$ with $\left|\phi^{E}\right\rangle=\sum_{m} \sqrt{p_{m}^{E}} \mathrm{e}^{i \psi_{m}}\left|\vec{v}_{m}\right\rangle$. Therefore, out-of-diagonal terms will not impose any additional constraints, and the optimal solution to the absent-minded driver's problem can always be chosen to be $\left|\phi^{E}\right\rangle=$ 
$\sum_{m} \sqrt{p_{m}^{E}} \mathrm{e}^{i \psi_{m}}\left|\vec{v}_{m}\right\rangle$ for a given vertex $\vec{p}^{E}$. A remarkable feature is that all the vertices except two, $(0, \ldots, 0)$ and $(1, \ldots, 1)$ - which represent the two possible deterministic strategies-, are implemented by means of entangled states.

The particular solution for a given pay-off assignment can be obtained in a straightforward manner by standard linear programming methods. Obtaining the set of possible optimal solutions can be significantly more difficult. For this purpose one can use a method developed in the context of the theory of $N$-party games. To every binary array $\vec{v}$, one can associate a subset of $P=\{1, \ldots, N\}, S_{v}=\{i: \vec{v}(i)=1\}$. The condition of indistinguishability on the arrays $\{\vec{v}\}$ is equivalent, in game-theoretical terms, to saying that the collection of sets, or coalition of players, $\left\{S_{v}\right\}$ is balanced. The concept of a minimally balanced collection $[16,17]$, which corresponds to the set of contributing arrays in a vertex of the polytope, has found important applications for the theory of $N$-party games. This theory does not apply to the absent-minded driver's problem; however, we can use an inductive method developed in that context [18] to generate the possible solutions (vertices) of the $N$-intersection problem from those of the $N-1$ problem.

Let us consider the absent-minded driver's problem with three intersections [2] and the set of pay-offs given in Fig. 2. In this case, the vertices of the polytope of possible solutions are $B_{1}=(0,0,0), B_{2}=(1,1,1), B_{3}=(0,0,1)+(1,1,0)$, $B_{4}=(0,1,0)+(1,0,1), B_{5}=(1,0,0)+(0,1,1), B_{6}=(0,0,1)+(0,1,0)+$ $(1,0,0)$, and $B_{7}=(0,1,1)+(1,0,1)+(1,1,0)$. It can be easily seen that, for any $0<n$, there is a probabilistic strategy that gives a higher expected pay-off than any deterministic one (see Fig. 3). More interestingly, there is a quantum entanglements-assisted strategy that gives a higher pay-off than any probabilistic or deterministic strategy (Fig. 3). For $0<n \leq 2$, the optimum strategy (pay-off 2) can be implemented by preparing three qubits in the socalled Greenberger-Horne-Zeilinger [14] state

$$
|\mathrm{GHZ}\rangle=\frac{1}{\sqrt{2}}(|001\rangle+|110\rangle)
$$

putting one qubit in each intersection and measuring the observable $\sigma_{z}$ defined in Eq. (3). For $2 \leq n \leq 8$, the optimum strategy (pay-off $(4+n) / 3$ ) can be implemented by preparing three qubits in the so-called $\mathrm{W}[19,20]$ state

$$
|\mathrm{W}\rangle=\frac{1}{\sqrt{3}}(|001\rangle+|010\rangle+|100\rangle),
$$

followed by the $\sigma_{z}$ measurement. Finally, for $n \geq 8$, the optimum pay-off (pay-off $n / 2$ ) can be reached in a similar manner with GHZ-type states, for 


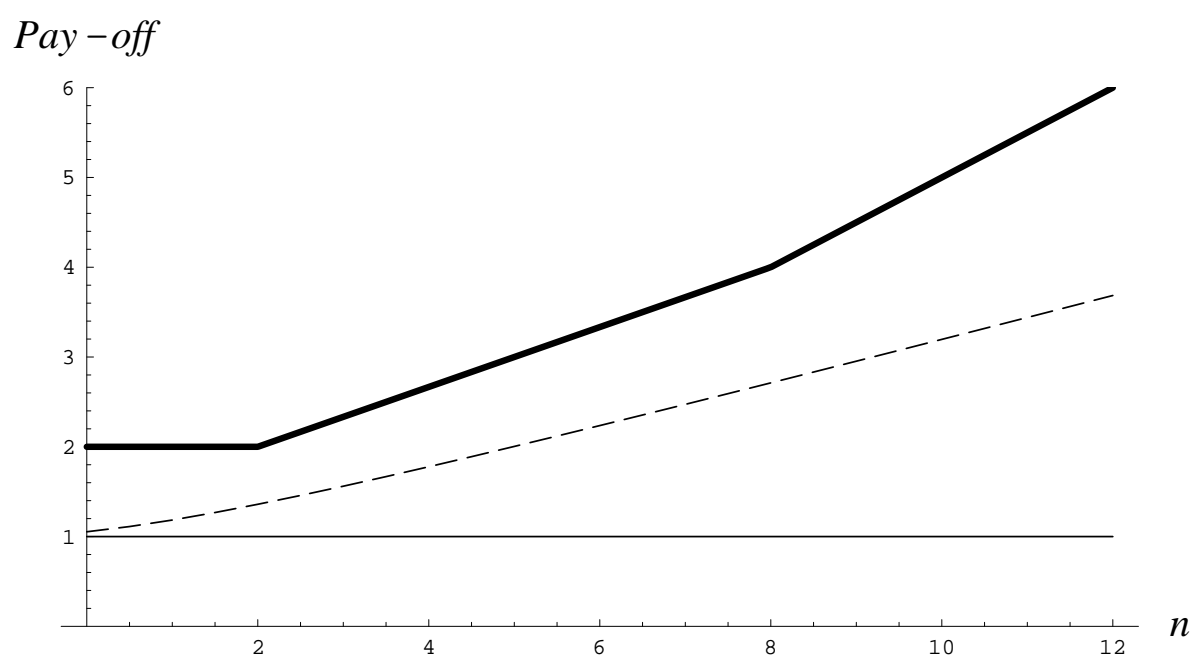

Fig. 3. Optimal expected pay-offs as a function of $n$ for deterministic (fine line, bottom), probabilistic (dashed line, in the middle), and quantum entanglement-assisted (thick line, top) strategies for the absent-minded driver's problem with three intersections of Fig. 2.

instance,

$$
\left|\mathrm{GHZ}^{\prime}\right\rangle=\frac{1}{\sqrt{2}}(|011\rangle+|100\rangle) .
$$

At the moment we do not have a characterization of the full set of optimal solutions for the $N$-intersection problem. From the previous reasoning we know that a necessary and sufficient condition for a solution to be optimal is that the contributing arrays are linearly independent. This also means that the superpositions will involve at most $N+1$ terms. We also know that, given a vertex (i.e., an optimal solution), if we exchange 0's for 1's and vice versa, then we have another vertex. From the previous examples it might seem that optimal solutions always involve equally weighted superpositions. However, for $N>3$ one can find optimal solutions for which this is not the case.

\section{Conclusions}

To sum up, the absent-minded driver's problem is that he does not know which intersection he is at and cannot have instructions indicating the route home. However, the assumptions under this scenario do not keep him from using certain quantum superpositions of instructions. We have seen that the use of entangled states allows him to increase the expected pay-off beyond what was previously considered the maximum pay-off.

The key point to understand the quantum advantage is that the limited absent- 
mindedness of the driver requires even-in-principle indistinguishability of the intersections; otherwise one cannot exclude the possibility that the driver possesses some information of the state of the rest of the universe (i.e., by prearranging it before his trip) such that during his trip he can figure out what intersection he is at. Hence, classically correlated physical systems (and specially anti-correlated systems) that could provide optimal expected pay-offs, are not allowed for decision-making. However, quantum states such as (4), (7), (8), (9), or those needed to implement all non-trivial vertices guarantee indistinguishability by fundamental laws of physics, while providing at the same time the desired correlations. According to quantum mechanics the quantum state of the $N$-qubit system is the most complete description of the system, in the sense that it provides the maximum possible information about any future experiments on these qubits. The proposed entangled states, hence guarantee that the absent-minded driver cannot determine at what intersection he is during his trip. In addition, these states hide the "correct" instruction sets in superpositions with non-optimal instructions, and thus provide high payoff values, without transgressing the assumptions behind the absent-minded driver's scenario.

The above examples suggest that strategies based on quantum entanglement can also provide advantages over classical strategies in other decision problems involving memory limitations or imperfect recall $[1,2,3]$ in which the indistinguishability of the alternatives plays an essential role.

\section{Acknowledgments}

We thank R. Raussendorf for useful discussions, O. Gühne for reading the manuscript, and the organizers of the Ninth Benasque Center for Science for support. A.C. acknowledges support from the Spanish Ministerio de Ciencia

y Tecnología Grant No. BFM2002-02815 and the Junta de Andalucía Grant No. FQM-239.

\section{References}

[1] M. Piccione, A. Rubinstein, Games and Economic Behavior 20 (1997) 3.

[2] R.J. Aumann, S. Hart, M. Perry, Games and Economic Behavior 20 (1997) 102.

[3] M. Piccione, A. Rubinstein, Games and Economic Behavior 20 (1997) 121.

[4] E. Klarreich, Nature 414 (2001) 244.

[5] S.J. van Enk, R. Pike, Phys. Rev. A 66 (2002) 024306. 
[6] D.A. Meyer, Phys. Rev. Lett. 82 (1999) 1052; Phys. Rev. Lett. 84 (2000) 790.

[7] J. Eisert, M. Wilkens, M. Lewenstein, Phys. Rev. Lett. 83 (1999) 3077; Phys. Rev. Lett. 87 (2001) 069802.

[8] S.C. Benjamin, P.M. Hayden, Phys. Rev. Lett. 87 (2001) 069801; Phys. Rev. A 64 (2001) 030301.

[9] R. Kay, N.F. Johnson, S.C. Benjamin, J. Phys. A 34 (2001) L547.

[10] J. Du, H. Li, X. Xu, M. Shi, J. Wu, X. Zhou, R. Han, Phys. Rev. Lett. 88 (2002) 137902.

[11] C.F. Lee, N.F. Johnson, Phys. World 15 (2002) 25; Phys. Rev. A 67 (2003) 022311.

[12] F. Guinea, M.A. Martín-Delgado, J. Phys. A 36 (2003) L197.

[13] J.S. Bell, Physics 1 (1964) 195.

[14] D.M. Greenberger, M.A. Horne, A. Zeilinger, in: M. Kafatos (Ed.), Bell's Theorem, Quantum Theory, and Conceptions of the Universe, Kluwer, Dordrecht, 1989, p. 69.

[15] J.A. Wheeler, in: A.R. Marlow (Ed.), Mathematical Foundations of Quantum Theory, Academic Press, New York, 1978, p. 9.

[16] H.E. Scarf, Econometrica 35 (1967) 50.

[17] L.S. Shapley, Nav. Res. Logist. Q. 14 (1967) 453.

[18] B. Peleg, Nav. Res. Logist. Q. 12 (1965) 155.

[19] I. Pitowsky, Phys. Lett. A 156 (1991) 137.

[20] W. Dür, G. Vidal, J.I. Cirac, Phys. Rev. A 62 (2000) 062314. 